\title{
Zur Rolle nichtmedizinischer Experten in der Medizin
}

Helmut Knolle

Korrespondenz:

PD Dr. Helmut Knolle Oberwohlenstrasse 20 CH-3033 Wohlen b. Bern

h.knolle@gmx.ch
Biologen, Chemiker und Physiker sind heute in allen grösseren Spitälern und medizinischen Forschungseinrichtungen anzutreffen, und kein Arzt würde es sich mit gutem Gewissen zutrauen, neue Wege zum Beispiel in der Strahlentherapie ohne den Rat eines Physikers zu beschreiten. Ganz anders verhalten sich Mediziner, wenn sie auf Fragen stossen, die in die Domäne der Mathematik führen. In solchen Fällen, die zugegebenermassen nicht sehr häufig sind, besteht die Tendenz, dem Problem auszuweichen oder ohne den Beistand eines Experten eine Lösung zu suchen. Um Missverständnisse zu vermeiden, sei schon jetzt gesagt, dass es hier nicht um medizinische Statistik geht. Die Probleme, von denen berichtet werden soll, haben nichts zu tun mit den typischen Fragestellungen dieser Disziplin, deren Nützlichkeit heute allgemein anerkannt ist. Sie betreffen zwei ganz verschiedene Gebiete: die Zellkinetik von Tumoren und die Ausbreitungsdynamik ansteckender Krankheiten. Die Ausführungen beschränken sich auf die Mitteilung der persönlichen Erfahrungen des Autors, der Mathematiker ist und mehr als zwanzig Jahre lang in medizinischen Projekten gearbeitet hat, aber sie verweisen auch auf Mängel des institutionellen Rahmens, in dem Forschung auf Randgebieten der Medizin in der Schweiz und in Deutschland stattfindet.

\section{Zellkinetik}

Das Studium der quantitativen Aspekte des Zellzyklus und des Wachstums von Zellpopulationen erlebte in den 1960er Jahren einen starken Aufschwung. Durch die In-vitro-Injektion einer geringen Dosis radioaktiven Thymidins in Versuchstiere oder Zellkulturen war man in der Lage, Zellen in der S-Phase radioaktiv zu markieren. Nach Entnahme eines Spezimens der interessierenden Zellpopulation und Anfertigung einer Autoradiographie konnte man den Anteil der markierten Zellen, den Markierungsindex, bestimmen. Die Weiterentwicklung dieser Technik erlaubte es ab 1966, den Anteil der proliferierenden Zellen (die Wachstumsfraktion) sowie die Dauer des Zellzyklus und der einzelnen Phasen zu schätzen. Die neuen Methoden und ihre wichtigsten Anwendungen in der experimentel-

\section{Experts non-médecins: leur rôle en médecine}

Aujourd'hui, les biologistes, chimistes et physiciens font partie du quotidien de tous les grands hôpitaux et centres de recherche médicale et aucun médecin ne voudra le cœur léger entreprendre de nouveaux pas par exemple en radiothérapie en se passant des conseils d'un physicien. Mais les médecins ont un tout autre comportement quand ils se heurtent à des questions qui mènent dans le domaine des mathématiques. Dans ces cas-là qui, nous concédons, ne sont pas très courants, la tendance est d'éviter le problème ou de lui chercher une solution sans recourir à un expert. Afin d'éviter tout malentendu, nous nous hâterons de dire qu'il ne s'agit pas ici de statistique médicale. Les problèmes dont il est question ici n'ont rien à faire avec les questions typiques de cette discipline dont I'utilité est aujourd'hui universellement reconnue. Ils concernent deux domaines bien distincts: la cinétique cellulaire de tumeurs et la dynamique d'expansion des maladies contagieuses. L'article se limite à la communication des expériences personnelles de l'auteur, mathématicien, qui a travaillé plus de vingt ans au service de projets médicaux. Ses propositions dénoncent aussi les lacunes du cadre institutionnel dans lequel a lieu la recherche dans les disciplines limitrophes de la médecine en Suisse et en Allemagne.

len Tumorforschung wurden von Gordon Steel in einer 1977 erschienenen Monographie umfassend dargestellt [1].

Es war naheliegend, die neuen zellkinetischen Methoden und Erkenntnisse auch für die Krebstherapie fruchtbar zu machen. Dies wurde in den 70er Jahren in der Tat versucht. Ein Konzept dieser Art ist unter dem Namen Synchroni- 
sationstherapie bekannt geworden. Dabei wurde zuerst der Mitosehemmer Vincristin gegeben, um die Tumorzellen zu synchronisieren, und nach dem Ende der Blockade in einem Moment, in dem man die synchronisierten Zellen in der S-Phase vermutete, eine Dosis eines Antimetaboliten, der selektiv Zellen in der S-Phase tötet. Es zeigte sich jedoch in klinischen Studien, dass die neue Therapie, so wie sie damals praktiziert wurde, nur die Nebenwirkungen verstärkte, ohne die Wirkung auf den Tumor zu verbessern.

Dies war der Stand der Dinge, als das deutsche Bundesministerium für Forschung und Technologie (BMFT) die Finanzierung des Projekts «Entwicklung und Testung von Arzneien für die Krebstherapie» bewilligte. An diesem Projekt waren insgesamt etwa 25 Wissenschaftler beteiligt, darunter Pharmakologen, experimentelle Krebsforscher vom DKFZ in Heidelberg und therapeutische Onkologen der Universitätsspitäler Köln, Essen-Bochum und Münster. Die medizinstatistische Begleitung des Projekts oblag dem Leiter des Instituts für Medizinische Biometrie der Medizinischen Hochschule Hannover, Prof. Dr. Berthold Schneider. Der Haushaltsplan des Projekts hatte ausserdem eine befristete Stelle für einen weiteren Mathematiker vorgesehen. Als ich Schneider im März 1980 an einer Tagung kennenlernte, bot er mir diese Stelle an, und ich sagte zu.

Im Juli 1980 nahm ich in Hannover die Arbeit auf und folgte Schneiders Vorschlag, die zellkinetischen Aspekte der Krebstherapie mit mathematischen Modellen zu untersuchen. Modelle für ungestörte Zellpopulationen gab es bereits, u. a. das Modell von Takahashi [2]. Deshalb konzentrierte ich mich zunächst darauf, die Durchführung und Auswertung der üblichen zellkinetischen Experimente zu verstehen und Angaben über zellkinetisch relevante Wirkungen von Zytostatika aus der Literatur zu sammeln. Dazu musste ich mich in ein für mich völlig neues Gebiet einarbeiten. Nach einiger Zeit konnte ich die biologischen Zusammenhänge einigermassen durchschauen, aber paradoxerweise konnte ich oft die Autoren gerade dann nicht verstehen, wenn sie rechneten. Ausserdem fand ich einige Male zum gleichen Thema verschiedene Arbeiten, die sich widersprachen. In einem Fall - es ging um die blockierende Wirkung von Cytarabin - gelang es mir, die Ursache des Irrtums in einem Denkfehler zu lokalisieren, der einem Autor in dem von G. Hartwich herausgegebenen Sammelband «Synchronisationsbehandlung maligner Tumoren» unterlaufen war $[3,4]$.
Ein anderes Problem, das die meisten zellkinetisch orientierten Onkologen in den 70er Jahren nicht lösen konnten, bestand vereinfacht gesagt darin, dass ihre Formeln nur für stationäre und langsamwachsende Zellpopulationen gültig waren. Aber wenn es um schnellwachsende Tumoren geht, kommt die Exponentialfunktion ins Spiel, und damit konnten nur wenige Onkologen umgehen. Durch einen in der Mathematik geläufigen Kunstgriff fand ich neue Formeln für die Zellkinetik, die leicht zu handhaben und trotzdem hinreichend genau sind. Diese publizierte ich 1984 in zwei kurzen Mitteilungen in der Zeitschrift «Cell and Tissue Kinetics» $[5,6]$. Gleichzeitig begann ich mit dem Aufbau eines umfangreichen Computerprogramms, dessen Zweck die Simulation der Chemotherapie eines Tumors sein sollte, und suchte noch intensiver in der Literatur nach Angaben über zellkinetische Parameter von Tumoren. Ich war allerdings schon skeptisch geworden und ging daran, die mathematische Stimmigkeit der Angaben in jedem einzelnen Fall zu überprüfen. Das Ergebnis war für mich schockierend. Bei einem Drittel der 65 untersuchten Tumoren wich der Wert der Wachstumsfraktion oder des Zellverlustfaktors um mehr als 20\% von dem Wert ab, den ich mit meiner Methode berechnen konnte [7]. Ich zog daraus den Schluss, dass es verfrüht wäre, das Konzept der Synchronisationstherapie wegen der enttäuschenden Ergebnisse des ersten Anlaufs ganz zu verwerfen. Denn es wäre ja denkbar, dass mangelnde begriffliche Klarheit und fehlerhafte Berechnungen die Ursache der Fehlschläge gewesen sind.

In meiner Habilitationsschrift, die ich im Sommer 1985 abschloss, widmete ich je ein Kapitel den mathematischen Modellen von Zellpopulationen, der experimentellen und rechnerischen Bestimmung von zellkinetischen Parametern und den zellkinetischen Vorgängen bei der Chemotherapie von Tumoren. Sie erschien 1988 im Springer-Verlag als Buch [8]. Der Rezensent von «Oncology» schrieb: «This book is an excellent introduction to practically oriented cell kinetic modelling and the methods suitable to determine the cell kinetic parameters» [9]. Dieses erfreuliche Resultat meiner Arbeit kontrastiert jedoch mit der bedauerlichen Tatsache, dass sich aus meiner fünfjährigen Arbeit in dem BMFT-Projekt kein wissenschaftlicher Kontakt mit irgendeinem der beteiligten Mediziner ergeben hat. Wo immer ich bei Onkologen anfragte, sagte man mir: Synchronisationsbehandlung und Zellkinetik sind passé. Vergeblich wies ich auf die von mir gefundenen Fehler bei der An- 
wendung der zellkinetischen Konzepte hin. Die neue Generation von Onkologen erhoffte die Lösung des Krebsproblems von aggressiven Therapien auf der Intensivstation oder von der Pharmaindustrie, der man die Entwicklung von Wunderwaffen gegen den Krebs zutraute. Die Medikamente, die heute angewendet werden, sind aber keine Wunderwaffen, sie sind nur wunderbar teuer. Eine einjährige Behandlung mit dem Krebsmittel Glivec von Novartis kostet rund 40000 Franken [10].

\section{Modellierung der Aidsepidemie}

In dieser Situation schien es mir das beste, das Arbeitsgebiet zu wechseln. Die beginnende Aidsepidemie legte es nahe, sich mit diesem Thema $\mathrm{zu}$ befassen. Nach einer Zwischenstation in Tübingen, wo ich eine Zeitlang einen Assistenten des Biometrikers Prof. Dr. Klaus Dietz vertreten konnte und mich mit der Poliomyelitis und mit Aids befasste, fand ich im November 1989 eine befristete Anstellung am Bundesamt für Gesundheit (BAG) in Bern. Im BAG bestand damals eine sogenannte Zentralstelle für Aidsfragen, die für alle praktischen Fragen der Prävention und Therapie zuständig war. Die objektive Beobachtung der Epidemie war hingegen Sache der Sektion Epidemiologie, in die ich integriert war.

Mit dieser Sektion war damals auch der weltweit bekannte englische Mathematiker Norman Bailey, der nach seiner Tätigkeit als Leiter der Abteilung Statistische Methodologie der WHO in der Schweiz geblieben war, lose assoziiert. Bailey hatte bereits zuvor in zwei vom Nationalfonds geförderten Projekten mathematische Modelle für die Ausbreitung von Hepatitis und HIV/Aids entwickelt. Sein Aidsmodell wurde nun in gemeinsamer Arbeit an die Situation in der Schweiz angepasst [11]. Wir verloren jedoch viel Zeit, weil die Zentralstelle für Aidsfragen uns drängte, den im ersten Halbjahr 1990 beobachteten Rückgang der neuen Aidsfälle gegenüber dem Vorjahr zu interpretieren. Es war offensichtlich ein grosser Wunsch der Zentralstelle, von uns eine Bestätigung des Erfolgs der von ihr ergriffenen Massnahmen zu erhalten. Dabei war doch wegen der langen Inkubationszeit von Aids auch ohne jede Modellrechnung klar, dass die Stop-Aids-Kampagne, die 1987 begonnen hatte, nach weniger als drei Jahren noch keine Wirkung auf die Zahl der Aidsfälle haben konnte. Dass die Präventionskampagne des BAG tatsächlich ein Erfolg war, hätte durch eine genaue Analyse der Daten des HIV-Meldewesens (mit Einbezug des Problems der Wiederholungstests und der Dunkelziffer) bewiesen werden müssen. Diese Analyse, die ich wegen der Arbeit an ande- ren Projekten erst 1997 durchführen konnte, ergab eine Halbierung der Neuansteckungen in der Periode 1990-1992 gegenüber der Periode 19851989 [12].

\section{Die Impfung gegen Kinderlähmung}

In Tübingen hatte mich Klaus Dietz auf die Paradoxien der Epidemiologie der Poliomyelitis und den 1988 gefassten Beschluss der WHO zur globalen Ausrottung dieser Krankheit aufmerksam gemacht. In den Jahren danach hatte ich diese Problematik nie ganz aus den Augen verloren. Zunächst legte ich Beweise für die lange Zeit umstrittene These vor, dass Polioviren auch durch das Trinkwasser übertragen werden [13]. In Deutschland und in der Schweiz war seit 1960 mit der aus attenuierten Lebendviren bestehenden oralen Poliovakzine (OPV) von Sabin geimpft worden. Diese hat den Vorteil, dass sie auch die stille Infektion mit Polioviren verhindert; ihr Nachteil ist, dass die Impfviren zu pathogenen Formen rückmutieren und auf Kontaktpersonen werden können. Als ich nun 1998 erfuhr, dass in Deutschland der Übergang von der OPV-Impfung zur ausschliesslichen Impfung mit der inaktivierten Poliovakzine (IPV) vollzogen wurde, fragte ich im BAG, was man in der Schweiz zu tun gedenke. Die Antwort, die ich zusammen mit einigen Rechtfertigungsartikeln aus deutschen Zeitschriften erhielt, lautete etwa, dass man keinen Grund sehe, dem deutschen Beispiel nicht zu folgen. Entsprechend wurde dann ein Jahr später gehandelt. Erst 2003 bot sich mir die Möglichkeit, zusammen mit einem Pharmakologen und einem Mediziner die Probleme, die sich für das globale Programm der WHO aus der Präferenz der entwickelten Länder für IPV ergeben, eingehend zu diskutieren. In einem gemeinsamen Artikel plädierten wir dafür, das reine IPV-Schema durch ein sequentielles Schema zu ersetzen, bei dem zuerst IPV und danach, wenn eine angeborene Immunschwäche ausgeschlossen werden kann, OPV gegeben wird. Die OPV-Dosis würde verhindern, dass IPV-geimpfte Besucher von Entwicklungsländern, in denen aus organisatorischen und finanziellen Gründen mit OPV geimpft werden muss, dort mit rückmutierten Impfviren infiziert werden und diese auf ungeimpfte Kontaktpersonen übertragen werden können [14]. Was man heute im BAG darüber denkt, wissen wir nicht, denn wir haben von keiner Reaktion erfahren.

\section{Sparen am falschen Ort}

Die letzte Bemerkung soll nicht falsch verstanden werden. Sie ist keine persönliche Kritik, sondern Kritik am Personalmangel und vielleicht an 
den Organisationsstrukturen im BAG. Norman Bailey konnte seinerzeit, als er als externer Berater für das BAG tätig war, stundenlange Gespräche mit den für das Aidsmeldewesen zuständigen Personen führen, bevor er dem Aidsmodell für die Schweiz den letzten Schliff gab. Das wäre heute unmöglich, weil die Sachbearbeiter schon mit der täglichen Routinearbeit überlastet sind. Auch heute sind externe Berater für das BAG tätig, aber das sind keine Experten in der Medizin oder irgendeinem für das Gesundheitswesen relevanten Gebiet, sondern Vertreter einer abstrakten Managementtheorie, deren Rezepte angeblich zur Steigerung der Effizienz beitragen. Ob damit die Arbeit des BAG qualitativ verbessert wird, muss doch sehr bezweifelt werden.

Dass der Personalabbau in der Bundesverwaltung unter Umständen erst recht zu höheren Kosten führen kann, möchte ich am Beispiel des Ankaufs von 3 Millionen Dosen Pockenimpfstoff darlegen. Schon 1999 erschienen in den USA die ersten Artikel in wissenschaftlichen Zeitschriften über die möglichen Folgen einer terroristischen Freisetzung von Pockenviren. Zwei Jahre später entstand in den Centers for Disease Control and Prevention eine Studie, die für die USA die vorbeugende Lagerung von 40 Millionen Dosen Pockenimpfstoff empfahl. Aber 2003 erschien dann eine Arbeit des Biomathematikers Martin Eichner vom Institut für Medizinische Biometrie der Universität Tübingen, die den amerikanischen Studien widersprach und $\mathrm{zu}$ dem Schluss kam, dass ein Pockenausbruch auch ohne Massenimpfung eingedämmt werden könne [15]. Das BAG hatte aber keinen Experten, der in der Lage gewesen wäre, angesichts dieser widersprüchlichen Positionen eine rationale Entscheidung vorzubereiten. Deshalb glaubte es, den Weg des geringsten Risikos gehen zu müssen, und kaufte 3 Millionen Dosen Impfstoff. Inzwischen hat jedoch das BAG im Hinblick auf die Möglichkeit einer Grippepandemie einen Beratervertrag mit Eichner abgeschlossen. Offenbar hat man, wenn auch sehr spät, eingesehen, dass die rationale Planung von Massnahmen gegen Epidemien auf mathematische Instrumente nicht verzichten kann.

\section{Literatur}

1 Steel G. Growth Kinetics of Tumours. Oxford: Clarendon Press; 1977.

2 Takahashi M. Theoretical basis for cell cycle analysis. J Theor Biol. 1966;13:202-11.

3 Knolle H. Kritischer Vergleich von einigen Arbeiten zum Blockierungseffekt von Cytosinarabinosid (Cytarabin). Arzneimittelforschung. 1983; 33(10):1507-9

4 Hartwich G (Hrsg.). Synchronisationsbehandlung maligner Tumoren. Erlangen: Perimed; 1976.

5 Knolle H. A simple method to calculate the cell-loss factor. Cell Tissue Kinet. 1984;17(3):311-2.

6 Knolle H. New formulae for the evaluation of a single set of data from double labelling with [14C]TdR and [3H]TdR. Cell Tissue Kinet. 1984;17(6):661-6.

7 Knolle H. Values of growth fraction and cell loss factor of sixty-five tumours re-examined. Cell Tissue Kinet. 1986;19:503-9.

8 Knolle H. Cell Kinetic Modelling and the Chemotherapy of Cancer. Berlin: Springer; 1988.

9 Eckhardt S (Rezension). Oncology. 1990;47(2).

10 Law J. Big Pharma. Das internationale Geschäft mit der Krankheit. Düsseldorf: Patmos; 2007.

11 Bailey NTJ. An improved hybrid HIV/AIDS model geared to specific public health data and decision making. Math Biosciences. 1993;117:221-37.

12 Knolle H, Minder C. Schätzung der HIV-Prävalenz in der Vergangenheit durch Zusammenführen von HIV- und AIDS-Daten. ISPM der Universität Bern; 1997.

13 Knolle H. Übertragung der Poliomyelitis durch Trinkwasser. Gesundheitswesen. 1995;57:351-4.

14 Knolle H, Egli A, Candrian U. Die Perspektive der globalen Ausrottung der Poliomyelitis. Gesundheitswesen. 2004;66:1-6.

15 Eichner M. Case isolation and contact tracing can prevent the spread of smallpox. Am J Epidemiol. 2003;158:118-218. 